\title{
Morphogen-Lineage Selector Interactions During Surface Epithelial Commitment
}

Sandra P. Melo ${ }^{1,3 *}$, Jillian M. Pattison ${ }^{1 *}$, Samantha N. Piekos ${ }^{1 *}$, Jessica L. Torkelson ${ }^{1}$, Elizaveta

Bashkirova ${ }^{1,4}$, Maxwell R. Mumbach ${ }^{1,2}$, Charlotte Rajasingh ${ }^{1}$, Hanson Hui Zhen ${ }^{1}$, Lingjie Li ${ }^{1}$, Eric Liaw $^{1,5}$, Daniel Alber ${ }^{1}$, Adam J. Rubin ${ }^{1}$, Gautam Shankar ${ }^{1}$, Howard Y. Chang ${ }^{1,2}$, Paul A. Khavari $^{1}$, and Anthony E. Oro ${ }^{1}$

${ }^{1}$ Program in Epithelial Biology, Stanford University School of Medicine, Stanford, CA 94305, USA. ${ }^{2}$ Center for Personal Dynamic Regulomes, Stanford, CA 94305, USA.

Current Addresses: ${ }^{3}$ Agilent Technologies, Santa Clara, CA 95051, USA. ${ }^{4}$ Columbia Stem Cell Initiative, Columbia University Medical Center, New York, NY 10032, USA. ${ }^{5}$ David Geffen School of Medicine at UCLA, Los Angeles, CA 90095, USA.

*These authors contributed equally to this work.

Address correspondence to: Anthony E. Oro oro@stanford.edu 
Human embryonic stem cell (hESC) differentiation promises advances in regenerative medicine ${ }^{1-3}$, yet conversion of hESCs into tissues such as keratinocytes requires a better understanding of epigenetic interactions between the inductive morphogens retinoic acid (RA) and bone morphogenetic protein 4 (BMP), and the master regulator $p 63^{4,5}$. Here we develop a robust, defined, keratinocyte differentiation system, and use a multi-dimensional genomics approach to interrogate the contributions of the morphogens and lineage selector to chromatin dynamics during early surface ectoderm commitment. In stark contrast to other master regulators ${ }^{6-9}$, we find using $p 63$ gain and loss of function hESC lines, that p63 effects major transcriptional changes only after morphogenetic action. Morphogens alter chromatin accessibility and histone modifications, establishing an epigenetic landscape for p63 to modify. In turn, p63 closes chromatin accessibility and promotes the accumulation of repressive $\mathrm{H} 3 \mathrm{~K} 27 \mathrm{me} 3$ histone modifications at sites distal to where it binds. Surprisingly, cohesin HiChIP ${ }^{10}$ visualization of genome-wide chromosome conformation reveals that both p63 and the morphogens contribute to dynamic long-range genomic interactions that increase the probability of negative transcriptional regulation at p63 target loci. p63-regulated accessibility, not H3K27me3 deposition, appears to drive early transcriptional changes. We illustrate morphogen-selector interactions by studying p63 negative feedback regulation of TFAP2C ${ }^{11}$, whereby disruption of the single p63 binding site results in a loss of p63mediated transcriptional control and dramatic increases in TFAP2C and p63 expression. Our study reveals the unexpected dependency of p63 on morphogenetic signaling to control long-range chromatin interactions during tissue specification and provides novel insights into how master regulators specify diverse morphological outcomes.

\section{Published protocols of hESC-derived keratinocytes suffer from heterogeneity due}

to feeders and additive variability ${ }^{5,12-15}$, thus we developed a xeno-free, chemicallydefined differentiation system based on E6 media ${ }^{16}$ supplemented with two morphogens, RA and BMP4 (Fig. 1a). This system was highly reproducible using hESCs and recapitulated commitment towards a surface ectoderm fate, indicated by immunofluorescence (IF) analysis of epithelial markers keratin $18(\mathrm{~K} 18)^{17}$ and $p 63^{18,19}$ by day 7 , followed by high levels of p63 and the keratinocyte maturation marker keratin 14 $(\mathrm{K} 14)^{20}$ by day 45 (Fig. 1a). Robust p63 expression occurred only when both morphogens were present, indicating a synergistic role for p63 accumulation (Fig. 1b,c, Extended Data Fig. 1). As morphogenetic exposure for 7 days induced both uniform p63 expression and subsequent keratinocyte development ${ }^{4,5}$, we interrogated this key 7 -day 
stage with a multi-dimensional genomics approach to understand the functional

39 interaction between p63 and the morphogens.

To assess the individual contributions to chromatin dynamics, we created p63 gain and loss of function hESCs using CRISPR/Cas9 technology (Fig. 1d,f) to yield a panel of four cell types: d0 (wild-type hESCs), d0 p63GOF (hESCs ectopically expressing p63), d7 p63WT (wild-type hESCs morphogen-treated, with endogenous p63), and d7 p63KO (hESCs morphogen-treated with no p63 expression). We verified

45 p63 expression in these cell lines through IF, western blot, and sequencing (Fig. 1e,g, 46 Extended Data Fig. 2).

Previous studies indicate that p63 overexpression can drive surface ectoderm

48 commitment ${ }^{21}$, yet remarkably, expression of p63 in hESCs was insufficient to induce differentiation (Fig. 1e, Extended Data Fig. 2). Consistent with this observation, transcriptome analysis using RNA-sequencing (RNA-seq) revealed moderate changes in expression in roughly 300 genes between d0 and d0 p63GOF cells, whereas more than

522400 genes were differentially expressed in d7 p63WT vs. d7 p63KO cells (Fig. 1h).

53 Further, independent of the presence or absence of p63, morphogen exposure resulted

54 in an exit from pluripotency and was required for p63 regulation of key transcription 55 factors associated with epithelial development (Fig. 1h, Extended Data Fig. 2c). These 56 important epithelial transcription factors, including TFAP2C, KLF4, GATA3, GRHL2,

57 MSX2, and ELF3, were all repressed by p63 upon morphogen treatment. We conclude 58 that morphogenetic signaling promotes a simple epithelial state, while enabling p63 to 59 modify the morphogen-induced transcriptome to drive these stratified epidermal fates. The striking influence of morphogens on p63 activity led us to investigate

61 whether differences in p63 genomic occupancy accounted for the altered transcriptional 62 activity. p63 ChIP-seq in d0 p63GOF and d7 p63WT revealed 7,960 and 6,097 p63

63 binding sites, respectively, and the p63 motif was significantly enriched under peaks in 
64 both datasets (Fig. 1i). Remarkably, over $70 \%$ of the sites were identical between both

65 datasets (Fig. 1j,k), while $17 \%$ of peaks were gained in the d0 p63GOF (Extended Data

66 Fig. 3a). Thus, differences in p63 occupancy cannot explain the dramatic morphogen-

67 regulated p63 activity.

We next characterized how the morphogens and p63 affected chromatin accessibility and deposition of four histone modifications (H3K27ac, H3K27me3, H3K4me3, H3K4me1) using the Assay for Transposase Accessible Chromatin followed by sequencing (ATAC-seq) and histone chromatin immunoprecipitation (ChIP-seq),

72 respectively. Overall, approximately 20,000 transposase accessible sites changed

73 during the induction phase, with 14,000 opening and 6,000 closing between d0 and d7

74 p63WT (Fig. 2a). Additionally, over one third of the morphogen-dependent accessible

75 sites became even more accessible upon p63 loss (Fig. 2a,d). Comparison of

76 established histone modifications in d7 p63WT vs. p63KO revealed significant

77 differences in $\mathrm{H} 3 \mathrm{~K} 27 \mathrm{me} 3$, yet no observable differences on activating promoter or

78 enhancer marks (Extended Data Fig. 3). Opposite to ATAC-seq changes, p63 absence

79 resulted in a significant decrease in signal of the H3K27me3 mark, whereas H3K27me3

80 increased in d0 p63GOF cells (Fig. 2b,d). ChromHMM analysis indicated most of the

81 accessibility changes and p63 binding sites occur in enhancers, rather than promoters

82 (Extended Data Fig. 3). We conclude that p63 edits a subset of the morphogen-induced

83 accessibility changes and regulates the accumulation of repressive H3K27me3 histone

84 modifications.

Lineage selectors can act both directly on the epigenetic landscape at the site of

86 binding to alter accessibility or histone modification deposition, or indirectly at a

87 distance ${ }^{22}$. To determine how p63 acts, we intersected the p63-dependent H3K27me3

88 regions and morphogen-dependent accessible sites with p63 binding sites, revealing

89 that few of the p63 binding sites overlapped with either of these changing elements (Fig. 
2e). These data indicate that most of the p63 epigenetic regulatory action occurs distal to p63 binding. Interestingly, when we assigned p63 binding sites, morphogendependent accessible sites, and differential H3K27me3 regions to the nearest genes through GREAT, we found that these elements converge on a common gene set, despite each being distinct genomic regions (Fig. 2f, Extended Data Fig. 3).

To assess the connectivity and dynamics of the three-dimensional architecture between these distinct genomic regions, we employed cohesin HiChIP, a recent method analogous to $\mathrm{Hi}-\mathrm{C}^{10}$, in all four cell types. We identified high-confidence chromatin contacts with $10 \mathrm{~kb}$ resolution using $\mathrm{FitHiC}^{23}$ (Extended Data Fig. 4) and demonstrated that $53 \%$ of p63 ChIP-seq peaks in d7 p63WT cells participate in these chromatin connections (Fig. 3a). Additionally, we illustrated that most morphogen and p63dependent dynamic elements also participate in looping connections. Notably, only $34 \%$ of genes GREAT identified as having transcriptional start sites (TSSs) connected to p63 binding sites were verified by cohesin HiChIP, reinforcing the non-uniformity of the existing chromatin landscape (Extended Data Fig. 3,5).

For the 4,409 protein-coding, p63-dependent genes, we determined the connectivity of their TSSs to a p63 binding site (Fig. 3b), revealing that $13 \%$ of these genes were in direct contact with p63 via chromatin looping $\left(1^{\circ}\right)$ and $11 \%$ were in contact via an indirect connection through a morphogen-dependent accessible site or H3K27me3 element $\left(2^{\circ}\right)$. Although more complex conformations through multiple elements $\left(3^{\circ}\right)$ were detected, random simulation demonstrated that p63 was not connected to $\mathrm{p} 63$-dependent genes by $3^{\circ}$ connections at a frequency above random chance (FDR < 0.005); thus we focused on the $0^{\circ}, 1^{\circ}$, and $2^{\circ}$ p63 connections (Fig. $3 \mathrm{~b}$, Extended Data Fig. 6).

We further interrogated the correlation between p63 connection to the TSS and transcriptional regulation, finding that $\mathrm{p} 63$ connectivity was insufficient to regulate gene 
expression. However, both p63-dependent and -independent genes connected to a p63

117 site were involved in organ development and cell differentiation, consistent with known

118 p63 function (Fig. 3c) $)^{7,8}$. Additionally, the probability of transcriptional repression was

119 significantly higher at genes connected to p63 (Fig. 3d). d7 p63-independent genes

120 connected to p63 include keratinocyte differentiation genes whose expression becomes

121 p63-dependent later during keratinocyte maturation, including p63 itself, MAFB, JAG1,

122 ID2, and the Epidermal Differentiation Cluster (Extended Data Table 1) ${ }^{24-26}$. These data

123 suggest that p63 and morphogen-regulated chromatin connections foreshadow future

124 gene action. In all, we demonstrated that a large subset of the morphogen and p63-

125 dependent elements are physically connected at d7 (Fig. 3e), accounting for the ability of 126 p63 to regulate the epigenetic landscape at a distance.

Next, we determined the extent to which p63 and the morphogens influenced

128 connectivity (Fig. $3 f)$. In $1^{\circ}$ (middle panel) and $2^{\circ}$ (right panel) connections, contacts

129 between morphogen-dependent accessible sites and p63 binding sites were regulated

130 by both the morphogens and p63, with loss of p63 abolishing the connections, while

131 overexpression of p63 failed to enhance them. Conversely, p63-H3K27me3 and p63-

132 TSS interactions were enhanced by the morphogens and p63 overexpression, and

133 weakened by p63 loss (Extended Data Fig. 7). Finally, we determined that of the 3D-

134 conformations connecting p63 to a TSS, the connections to both morphogen-dependent

135 accessible sites (Fig. 3g) and TSSs demonstrated greater repression than p63

136 connected via an H3K27me3 peak (Extended Data Fig. 7). These findings indicate that

137 for optimal p63-regulated transcription both the morphogens and p63 are needed.

138 From our global analyses, we identified TFAP2C, a critical epithelial regulator ${ }^{11}$,

139 as a gene induced by morphogens and repressed by p63 that exhibits a complex

140 chromatin architecture driving its regulation. We sought to illustrate the p63-morphogen

141 interactions by dissecting the p63 negative feedback regulation of this key 
142 developmental regulator (Fig. 4). Cohesin HiChIP and genomic analysis at this locus

143 (Fig. 4a, Extended Data Fig. 8) revealed a distal p63 binding site with three d7 p63WT

144 connections to the TSS: through a direct contact, the adjacent morphogen-dependent

145 accessible site, and the distal H3K27me3 peak, all within $400 \mathrm{~kb}$. We confirmed our

146 cohesin HiChIP with UMI-4C ${ }^{27}$, a locus-specific technique, using primer viewpoints

147 around the three connections (Extended Data Fig. 9).

148 Comparison of the chromosome conformation among the different cell lines

149 indicated that $\mathrm{p} 63$ presence enhances connectivity to all three of the main loops at $\mathrm{d} 7$

150 and in the absence of p63, the connections and transcriptional output collapse.

151 Morphogen exposure connects p63 to the induced neighboring morphogen-dependent

152 accessible site, but the connection relies on ongoing p63 expression to maintain it, as

153 loss of p63 fails to uphold it despite morphogen presence. Thus, our analysis of the

154 TFAP2C locus shows that both the morphogens and p63 contribute to proper regulation.

To validate the importance of the morphogen-dependent accessible site, we

removed the region using CRISPR/Cas9 and demonstrated a loss of morphogen-

157 induced TFAP2C expression (Extended Data Fig. 8b). Furthermore, we hypothesized

158 that removal of the p63 binding site should drive both TFAP2C and p63 expression,

159 given our observation that TFAP2C induces p63 expression in hESCs (unpublished

160 results) and that p63 provides important early negative regulation of TFAP2C. To test

161 this, we deleted the p63 binding site (p63BSKO) and found dramatically elevated levels

162 of TFAP2C at d7, consistent with the predicted negative feedback modulation of

163 TFAP2C by p63 (Fig. 4b,c). Moreover, d7 p63BSKO cells showed increased expression

164 of p63, demonstrating the need for tight p63-morphogen regulation to control the levels

165 of key developmental factors. Histone ChIP-qPCR revealed a loss of H3K27me3

166 accumulation at both the TSS and the distal H3K27me3 site in d7 p63BSKO cells, while

167 other non p63-connected sites remained unaffected (Fig. 4d). Similarly, the morphogen- 
dependent accessible site became more accessible in d7 p63BSKO cells, to levels

169 found in $\mathrm{d} 7 \mathrm{p} 63 \mathrm{KO}$ cells (Fig. 4d), confirming the connectivity of these distal elements.

$170 \quad$ Here we deepen our understanding of the interplay between morphogens and

171 lineage selectors during surface ectoderm commitment, and find the surprising inability

172 for the lineage selector p63 to function in the absence of morphogen action. Morphogens

173 provide the powerful driving force for cell state change by inducing expression of the

174 lineage factor while also altering chromatin accessibility, histone modifications, and

175 chromosome conformation. p63, in turn, further modifies the morphogen-dependent

176 epigenetic landscape to drive surface ectoderm differentiation. Further, our results

177 illustrate how chromatin connections to the lineage selector p63 are necessary and more

178 likely to induce gene expression changes, but are not sufficient. Our finding that p63 at

$179 \mathrm{~d} 7$ is poised to act on later keratinocyte differentiation genes (Extended Data Table 1) ${ }^{24-}$

$180{ }^{26}$ suggests the existence of additional inductive influences after addition of RA/BMP that

181 will enable broader p63-dependent transcription. This is functionally similar to "poised"

182 histone modifications and provides a structural explanation of how the order of

183 morphogen exposure can determine downstream transcriptional programs. This study

184 has important implications for the apparent autonomy of lineage selectors and for the

185 basis of morphogenesis. Our work suggests that small changes in morphogen activity

186 can dramatically alter the induced chromosome landscape and connectivity, explaining

187 how a single lineage selector like p63 can direct such a panoply of transcriptional

188 programs depending on the specific morphogen exposure. 
191 1. Walmsley, G.G., et al. Induced pluripotent stem cells in regenerative medicine 192 and disease modeling. Curr Stem Cell Res Ther 9, 73-81 (2014).

193 2. Inoue, H., Nagata, N., Kurokawa, H. \& Yamanaka, S. iPS cells: a game changer

\section{References}

for future medicine. The EMBO journal 33, 409-417 (2014).

3. Hanna, J., et al. Treatment of sickle cell anemia mouse model with iPS cells generated from autologous skin. Science 318, 1920-1923 (2007).

4. Umegaki-Arao, N., et al. Induced pluripotent stem cells from human revertant keratinocytes for the treatment of epidermolysis bullosa. Sci Trans/ Med 6, 264 ra164 (2014).

5. Sebastiano, V., et al. Human COL7A1-corrected induced pluripotent stem cells for the treatment of recessive dystrophic epidermolysis bullosa. Sci Transl Med 6, 264ra163 (2014).

6. Zaret, K.S. \& Carroll, J.S. Pioneer transcription factors: establishing competence for gene expression. Genes \& development 25, 2227-2241 (2011).

7. Yang, A., et al. p63 is essential for regenerative proliferation in limb, craniofacial and epithelial development. Nature 398, 714-718 (1999).

8. Mills, A.A., et al. p63 is a p53 homologue required for limb and epidermal morphogenesis. Nature 398, 708-713 (1999).

9. Lupien, M., et al. FoxA1 translates epigenetic signatures into enhancer-driven lineage-specific transcription. Cell 132, 958-970 (2008).

10. Mumbach, M.R., et al. HiChIP: efficient and sensitive analysis of protein-directed genome architecture. Nature methods 13, 919-922 (2016).

11. Qiao, Y., et al. AP2gamma regulates neural and epidermal development downstream of the BMP pathway at early stages of ectodermal patterning. Cell research 22, 1546-1561 (2012). 
216 12. Metallo, C.M., Ji, L., de Pablo, J.J. \& Palecek, S.P. Retinoic acid and bone morphogenetic protein signaling synergize to efficiently direct epithelial differentiation of human embryonic stem cells. Stem cells 26, 372-380 (2008).

13. Itoh, M., et al. Generation of 3D skin equivalents fully reconstituted from human induced pluripotent stem cells (iPSCs). PloS one 8, e77673 (2013).

14. Guenou, H., et al. Human embryonic stem-cell derivatives for full reconstruction of the pluristratified epidermis: a preclinical study. Lancet $374,1745-1753$ (2009).

15. Coraux, C., et al. Reconstituted skin from murine embryonic stem cells. Curr Biol $13,849-853$ (2003).

16. Chen, G., et al. Chemically defined conditions for human iPSC derivation and culture. Nature methods 8, 424-429 (2011).

227 17. Owens, D.W. \& Lane, E.B. The quest for the function of simple epithelial keratins. BioEssays : news and reviews in molecular, cellular and developmental biology 25, 748-758 (2003).

18. Senoo, M., Pinto, F., Crum, C.P. \& McKeon, F. p63 Is essential for the proliferative potential of stem cells in stratified epithelia. Cell 129, 523-536 (2007).

19. Koster, M.I. \& Roop, D.R. Mechanisms regulating epithelial stratification. Annu Rev Cell Dev Biol 23, 93-113 (2007).

20. Green, H., Easley, K. \& luchi, S. Marker succession during the development of keratinocytes from cultured human embryonic stem cells. Proceedings of the National Academy of Sciences of the United States of America 100, 1562515630 (2003).

21. Aberdam, E., et al. A pure population of ectodermal cells derived from human embryonic stem cells. Stem cells 26, 440-444 (2008). 
241 22. Wamstad, J.A., et al. Dynamic and coordinated epigenetic regulation of

242 developmental transitions in the cardiac lineage. Cell 151, 206-220 (2012).

243 23. Ay, F., Bailey, T.L. \& Noble, W.S. Statistical confidence estimation for Hi-C data

244 reveals regulatory chromatin contacts. Genome research 24, 999-1011 (2014).

245 24. Barton, C.E., et al. Novel p63 target genes involved in paracrine signaling and

246 keratinocyte differentiation. Cell death \& disease 1, e74 (2010).

247 25. Koh, L.F., Ng, B.K., Bertrand, J. \& Thierry, F. Transcriptional control of late

248 differentiation in human keratinocytes by TAp63 and Notch. Exp Dermatol 24,

$249 \quad 754-760(2015)$.

250 26. Truong, A.B., Kretz, M., Ridky, T.W., Kimmel, R. \& Khavari, P.A. p63 regulates

251 proliferation and differentiation of developmentally mature keratinocytes. Genes

252 \& development 20, 3185-3197 (2006).

253 27. Schwartzman, O., et al. UMI-4C for quantitative and targeted chromosomal contact profiling. Nature methods 13, 685-691 (2016). 


\section{Acknowledgements}

257 We thank members of the Oro Laboratory, P. Greenside, J. Wysocka, A. Kundaje, O.

258 Wapinski, and D. Webster for helpful discussions and comments. This work was

259 supported by CIRM Tools grant RT3-07796 (A.E.O.), NIH/NIAMS grant F32AR070565

260 (J.M.P.), and NIH P50 HG007735 (H.Y.C.).

\section{Author Contributions}

263 S.P.M. and J.M.P. designed and executed experiments, analyzed data, and wrote the 264 manuscript. S.N.P. analyzed data and edited the manuscript. J.L.T., E.B., M.R.M., C.R., 265 H.H.Z., and L.L. executed experiments and contributed to experimental design. E.L., 266 D.A., A.J.R., and G.S. contributed to data analysis. H.Y.C. and P.A.K. contributed to 267 experimental design. A.E.O. designed experiments, analyzed data, wrote the 268 manuscript, and conceived the project with S.P.M.

\section{Author Information}

271 These authors contributed equally to this work.

272 Sandra P. Melo, Jillian M. Pattison, \& Samantha N. Piekos

274 Affiliations

275 Program in Epithelial Biology, Stanford University School of Medicine, Stanford,

276 California, USA

277 Sandra P. Melo, Jillian M. Pattison, Samantha N. Piekos, Jessica L. Torkelson, Elizaveta

278 Bashkirova, Maxwell R. Mumbach, Charlotte Rajasingh, Hanson Hui Zhen, Lingjie Li,

279 Daniel Alber, Adam J. Rubin, Gautam Shankar, Howard Y. Chang, Paul A. Khavari, \&

280 Anthony E. Oro 
282 Center for Personal Dynamic Regulomes, Stanford, CA 94305, USA

283 Maxwell R. Mumbach \& Howard Y. Chang

284

285 Current Addresses

286 Agilent Technologies, Santa Clara, CA 95051, USA

287 Sandra P. Melo

288

289 Columbia Stem Cell Initiative, Columbia University Medical Center, New York, NY

290 10032, USA

291 Elizaveta Bashkirova

292

293 David Geffen School of Medicine at UCLA, Los Angeles, CA 90095, USA

294 Eric Liaw

295

296 Corresponding Author

297 Correspondence to Anthony E. Oro: oro@stanford.edu

298

299 Competing Interests

300 The authors declare no competing financial interests. 


\section{Figure Legends}

302 Figure 1. Morphogens and the lineage selector p63 cooperate to drive early stratified 303 epithelial differentiation. (a) Differentiation of hESCs into keratinocytes takes 60 days in 304 the xeno-free, defined system. Treatment with RA and BMP4 for 7 days induces K18 305 and p63 expression. Switching the cells into keratinocyte media allows for selection and 306 growth of functional keratinocytes (kc) that express K14 and p63. (b,c) hESCs need 307 exposure to both RA and BMP4 to achieve high p63 expression. Error bars represent 308 standard deviation. (d) Strategy for generating the d0 p63GOF cell line. Numbered black 309 boxes signify exons. (e) Expression of p63 in the d0 p63GOF line, showing even with

310 Dox treatment, there is no loss in Oct4 expression. (f) Strategy for generating the p63KO

311 line, using a two gRNA CRISPR/Cas9 approach. (g) IF validation of the p63KO line,

312 showing loss of p63 expression and higher levels of K18. All IF scale bars represent 50

$313 \mu \mathrm{m}$. (h) Differential expression analysis from RNA-seq (measured by DESeq2) between

314 the $d 0$ and d0 p63GOF lines (upper panel), and the d7 p63WT and p63KO lines (lower 315 panel). The gray dots on the scatter plot represent no change in gene expression 316 between the two cell types, while red represents increased expression in the d0 or d7 317 wild type by a $>2$ fold change and blue represents decreased expression in the $\mathrm{d} 0$ or $\mathrm{d} 7$ 318 wild type by $a<-2$ fold change. Key transcription factors associated with epithelial 319 development are induced by the morphogens and repressed by p63 at d7. (i) The p63 320 motif was the most significantly recovered motif under p63 ChIP-seq peaks in d0 321 p63GOF and d7 p63WT cells. (j) p63 binds distal to TSSs as depicted at the HES1 locus

322 (70 kb away) and to the same sites in d0 p63GOF and d7 p63WT. (k) p63 binds to

323 similar sites genome-wide with and without morphogen presence, as depicted in the

324 Venn Diagram. The majority of the $\mathrm{d} 7 \mathrm{p} 63$ binding sites are fully recovered in the $\mathrm{d} 0$ 325 p63GOF line. 
327 Figure 2. The morphogens establish an epigenetic landscape that p63 modifies at a

328 distance. (a) Differential accessible regions between d0 and d7 p63WT as analyzed

329 using DESeq2 on ATAC-seq signal. Heatmaps represent the signal at these ATAC

330 regions within the various cell types and assays: p63 ChIP-seq signal (red, left panel),

331 ATAC-seq signal (blue, middle panel), and H3K27me3 ChIP-seq signal (purple, right

332 panel). 14,191 differential regions become more accessible upon morphogen treatment

333 (morphogen-dependent). (b) Differential H3K27me3 regions between d7 p63WT and

$334 \mathrm{p} 63 \mathrm{KO}$ as analyzed by DESeq2. Heatmaps represent the same datasets as (a) only

335 signal is shown at differential H3K27me3 sites (3,793 sites). (c) ATAC-seq (blue) and

336 H3K27me3 (purple) signal at p63 binding sites (red). (d) Signal intensities of p63 ChIP-

337 seq, ATAC-seq, and H3K27me3 ChIP-seq shown at the TFAP2A locus. (e) The overlap

338 of genomic regions that are differential as measured in (a), (b), and (c). The genomic

339 location intersect is very low. (f) GREAT analysis linking the above differential regions to

340 the closest gene shows that these elements converge on a similar gene set. While their

341 physical genomic locations do not overlap, they are linked to a common gene via

342 GREAT.

344 Figure 3. p63 - TSS connections are associated with negative regulation genome-wide.

345 (a) Number of p63 binding sites (BS), p63-dependent (p63-dep) H3K27me3 sites,

346 morphogen-dependent (morph-dep) ATAC sites, and p63-dependent TSSs that

347 participate in chromatin looping (Anchored, red) vs those that do not (Not Anchored,

348 blue) in d7 p63WT cells. (b) Percentage of p63-independent (p63-indep) genes (blue)

349 and p63-dep genes (red), whose TSS is connected to p63 by direct binding $\left(0^{\circ}\right)$, direct

350 contact $\left(1^{\circ}\right)$, or connected via one $\left(2^{\circ}\right)$ or two $\left(3^{\circ}\right)$ morph-dep ATAC and/or p63-dep

351 H3K27me3 elements. (c) Gene Ontological Terms associated with p63-indep genes

352 (blue) and p63-dep genes (red), which are connected to p63. (d) ecdf of the log2 fold 
change in gene expression between d7 p63WT vs d7 p63KO cells ( $d 7$ p63KO / d7

p63WT) for all p63 connected genes (red) and p63 $1^{\circ}$ connected genes (blue) compared

to all genes (black). (e) $1^{\circ}$ contact connections between p63 BS (red), p63-dep

356 H3K27me3 (gold), and morph-dep ATAC (blue). (f) Change in connectivity strength

357 between various cell types of all contacts (left panel), p63 - morph-dep ATAC contacts

358 (middle panel), and p63 - morph-dep ATAC contacts in which both elements are

359 connected to the TSS (right panel). $\mathrm{n}=$ number of contacts. $(\mathrm{g})$ ecdf of the change in

360 expression level ( $d 7$ p63WT vs d7 p63KO) of genes whose TSS is connected to a p63

361 BS and morph-dep ATAC site, which in turn are connected to each other (green)

362 compared to all genes (black). FDR by monte carlo simulation

$363{ }^{* *}$ FDR $<0.01,{ }^{* *}$ FDR $<0.001$. Angela-Darling k-samples test ${ }^{* * *} p<1 \times 10^{-10}$.

Figure 4. p63 negatively regulates TFAP2C expression through morphogen-induced

and p63-dependent distal elements and connectivity. (a) Cohesin HiChIP reveals complex looping interactions between numerous distal elements at the TFAP2C locus. Schematic for the morphogen and p63-dependent interactions (top panel) with virtual 4C plots of the normalized cohesin HiChIP data below (bottom panel). (b) The p63 binding site was deleted using CRISPR/Cas9 to determine the effects of its loss on TFAP2C expression. p63BSKO is a 520 bp deletion surrounding the p63 ChIP-seq peak. (c)

372 Deletion of the p63 binding site leads to an increase in TFAP2C expression similar to the 373 levels seen in the $\mathrm{d} 7 \mathrm{p} 63 \mathrm{KO}$ cells (NS - not significant). Loss of TFAP2C expression also 374 leads to a dramatic increase in p63 expression. Relative pixel intensity was calculated 375 from 3 independent images and scale bars represent $20 \mu \mathrm{m}$. (d) ChIP-qPCR for $376 \mathrm{H} 3 \mathrm{~K} 27 \mathrm{me} 3$ at the TFAP2C locus shows a decrease in the histone mark in the $\mathrm{d} 7$ 377 p63BSKO cells, similar to the d7 p63KO cells ( ${ }^{*} p-$ value $\left.<0.01\right)$. ATAC-qPCR at this 378 locus shows an increase in accessibility at the d7 ATAC peak in d7 p63BSKO, again 
bioRxiv preprint doi: https://doi.org/10.1101/348839; this version posted June 16, 2018. The copyright holder for this preprint (which was not certified by peer review) is the author/funder. All rights reserved. No reuse allowed without permission.

379 similar to the $d 7 \mathrm{p} 63 \mathrm{KO}\left({ }^{*} \mathrm{p}\right.$-value $\left.<0.05\right)$. Deletion of the $\mathrm{p} 63$ binding site results in a

380 loss of tightly controlled TFAP2C expression. Both graphs depict signal relative to input

381 and error bars represent standard error of the mean.

382 


\section{Methods}

\section{CRISPR/Cas9 guided genome editing}

385 gRNAs were designed using the online tool available at http://crispr.mit.edu/28, selected

386 based on the highest scores and the least off-targets, and incorporated into a DNA

387 fragment bearing all the components necessary for gRNA expression ${ }^{29}$. Donor

388 sequences were designed by selecting $700 \mathrm{bp}$ arms flanking left and right of the region

389 to be modified. Both gRNAs and donor sequences were synthesized as 5-

390 phosphorylated gene blocks (IDT) and cloned into a blunted plasmid with puromycin

391 selection, except for gRNAs targeting the AAVS1 locus, which were acquired through

392 Addgene (Plasmid \# 72833) ${ }^{30}$. The d0 p63GOF line was generated by integrating the

393 humanized p63 mouse cDNA under the control of a Tetracycline Responsive Element

394 (TRE) to the AAVS1 locus. Doxycycline (Sigma) was added to the media for 2 days at a

395 concentration of $2 \mathrm{ug} / \mathrm{ml}$ to induce expression of $\mathrm{p} 63 \mathrm{in} \mathrm{hESCs}$.

397 hESC culture and transfection

398 H9 human embryonic stem cells were cultured on Vitronectin Recombinant Human

399 Protein (Life Technologies) in Essential 8 medium (E8, Life Technologies) as described

400 previously ${ }^{16}$. Cells were passaged every three days as clumps with $0.5 \mathrm{mM}$ EDTA

401 (Lonza). For transfection, $2 \times 10^{6}$ cells were nucleofected using AmaxaTM P3 Primary

402 Cell 4D-Nucleofector (Lonza) as recommended by the manufacturer, with no more than

403 a $10 \mathrm{uL}$ mix of 2 ug of plasmid carrying each gRNA, 2 ug of plasmid carrying hCas 9 and

$404 \quad 2-4$ ug of plasmid carrying the donor DNA to repair the Cas9/gRNA induced break by

405 homologous recombination. Cells were plated and allowed to recover for a minimum of

406 6h in E8 media supplemented with 2 uM thiazovivin (Stemgent). Drug selection with 1

$407 \mathrm{ug} / \mathrm{mL}$ puromycin (InvivoGen) started 48h after transfection and lasted 2 days for loss of 
408 function cell lines, or continued for several days for gain of function cell line. Colonies

409 were picked 10 days after selection and genotyped by PCR to confirm homozygosity.

\section{In vitro epithelial hESC differentiation}

412 For differentiation, $6.2 \times 10^{3}$ cells $/ \mathrm{cm}^{2}$ were plated as colonies on Vitronectin coated 413 plates. Next day, media was changed to Essential 6 (E6, Life Technologies)

414 supplemented with 1 uM RA (Sigma) and 5 ng/mL Recombinant Human BMP-4 (R\&D

415 Systems), and replaced every two days for seven days, at which point cells were

416 dissociated with Accutase (StemCell Technologies) and collected for downstream

417 analysis, or media was replaced to Defined Keratinocyte-SFM media (DKSFM, Life

418 Technologies) for terminal differentiation into keratinocytes.

\section{Immunofluorescence staining}

421 Cells were cultured on glass cover slips in 12 wells, subjected to the appropriate

422 treatment and fixed for $10 \mathrm{~min}$ at room temperature in $4 \%$ paraformaldehyde in PBS.

423 Cells were permeabilized for $10 \mathrm{~min}$ with permeabilization buffer $(0.1 \%$ Triton-X $+0.05 \%$

424 Tween-20 in PBS) and blocked for 30 min with 10\% Horse Serum (Vector Laboratories)

425 in permeabilization buffer. Antibodies at appropriate dilutions were incubated overnight

426 at $4^{\circ} \mathrm{C}$. Secondary antibodies were added at 1:500 dilution and incubated at room

427 temperature protected from light for $1 \mathrm{~h}$. Cells were washed three times in Hoechst

428 1:10,000 in PBS, and glass cover slips were mounted onto glass slides with mounting

429 medium before imaging. Antibodies were diluted in permeabilization buffer at the

430 indicated dilutions: AP-2y (1:100, Cell Signaling 2320S), p63 (1:200, Genetex

431 GTX102425), KRT18 (1:800, R\&D AF7619), KRT14 (1:1000, BioLegend 906001), OCT4

432 (1: 100, BioLegend 631902). 


\section{RNA extraction and library preparation}

435 For RNA extraction, cells were lysed directly in Trizol (Invitrogen), purified as indicated

436 by the manufacturer, and then run through RNeasy columns (Qiagen). Libraries for

437 RNA-seq were prepared using TrueSeq RNA Library Prep Kit v2 (Illumina) according to

438 the manufacturer's protocol. Real time PCR was performed with SYBR Green PCR

439 master mix (Life Technologies) and in a Stratagene real time PCR machine.

441 Chromatin immunoprecipitation (ChIP) and library preparation

442 Cells were cross-linked in suspension for 10 min using freshly prepared $1 \%$

443 formaldehyde (Thermo Scientific) in PBS. Subsequently, glycine was added to a final

444 concentration of $0.125 \mathrm{M}$ to quench formaldehyde, and cells were washed twice with

445 cold PBS. $60 \times 10^{6}$ or $10 \times 10^{6}$ cross-linked cells were used per ChIP for p63 or histone

446 marks, respectively. Cells were lysed in lysis buffer (50 mM Tris-HCl pH 8.0, $10 \mathrm{mM}$

447 EDTA, $0.5 \%$ SDS, $1 \mathrm{X}$ protease inhibitors) for 30 minutes on ice and sonicated for $2 \mathrm{~h}$

448 using a Bioruptor (Diagenode) to achieve a chromatin size between 200 and $300 \mathrm{bp}$.

449 Chromatin was centrifuged to remove debris, quantified and diluted in dilution buffer (50

$450 \mathrm{mM}$ Tris-HCl pH 8.0, $10 \mathrm{mM}$ EDTA, $1 \mathrm{X}$ protease inhibitors) to achieve a $0.1 \%$ SDS final

451 concentration. Sheared chromatin was incubated overnight at $4^{\circ}$ with appropriate

452 antibodies, followed by incubation with $30 \mathrm{uL}$ of agarose $\mathrm{G}$ beads (Invitrogen) for $4 \mathrm{~h}$ at

$4534^{\circ} \mathrm{C}$. Antibodies were used at the indicated concentrations per ChIP (per $10 \times 10^{6}$ cells):

454 p63 (12 uL, Active Motif 39739), H3K4me3 (5 ug, Abcam ab8580), H3K4me1 (5 ug,

455 Abcam ab8895), H3K27Ac (5 ug, Abcam ab4729), H3K27me3 (5 ug, Millipore 07-449).

456 Beads were washed twice each with low salt buffer $(50 \mathrm{mM}$ Tris- $\mathrm{HCl} \mathrm{pH} 8.0,0.15 \mathrm{M}$

$457 \mathrm{NaCl}, 1 \mathrm{mM}$ EDTA $\mathrm{pH} 8.0,0.1 \%$ SDS, $1 \%$ triton $\mathrm{X}-100,0.1 \%$ sodium deoxycholate),

458 high salt buffer (50 mM Tris-HCl pH 8.0, $0.5 \mathrm{M} \mathrm{NaCl}, 1 \mathrm{mM}$ EDTA pH 8.0, 0.1\% SDS,

$4591 \%$ triton $\mathrm{X}-100,0.1 \%$ sodium deoxycholate $)$, and $\mathrm{LiCl}$ buffer $(50 \mathrm{mM}$ Tris- $\mathrm{HCl} \mathrm{pH} 8.0$, 
461 was eluted in $100 \mathrm{uL}$ of elution buffer (50 $\mathrm{mM} \mathrm{NaHCO}_{3}, 1 \% \mathrm{SDS}$ ) and crosslinks were

462 reversed with $4 \mathrm{uL}$ of $5 \mathrm{M} \mathrm{NaCl}$ incubated overnight at $67^{\circ} \mathrm{C}$. RNA was removed by

463 adding $1 \mathrm{uL}$ of $10 \mathrm{mg} / \mathrm{mL}$ RNase $\mathrm{A}$ and incubating for $30 \mathrm{~min}$ at $37^{\circ} \mathrm{C}$. DNA was cleaned

464 using the Qiagen Qiaquick PCR purification kit and quantified using Qubit (Invitrogen).

465 Between 5 and 10 ng of pooled DNA were used for library preparation using NEBNext kit

466 (New England Biolabs) and Agencourt AMPure XP beads (Beckman) according to the

467 manufacturer's protocol. Single-read libraries were sequenced on Illumina NextSeq

468 sequencer.

\section{Assay for Transposase Accessible Chromatin (ATAC-seq)}

471 ATAC-seq was performed as described ${ }^{31}$. Briefly, after treatment with Accutase, $7 \times 10^{4}$

472 cells were washed with cold PBS and lysed using $0.1 \%$ NP40 in RSB buffer. Nuclei

473 pellets were Tn5 transposed using the DNA Sample Preparation Kit from Nextera®.

474 Libraries were amplified for 9-15 total cycles using the Ad1 and Ad 2.1-2.16 barcodes.

475 Libraries were purified using the Min-Elute columns (Qiagen) and eluted with $10 \mu \mathrm{L}$ of

476 buffer EB. Library DNA concentrations were determined with Bioanalyzer High-

477 Sensitivity DNA analysis kit (Agilent). Paired-end libraries were sequenced initially on a

478 MiSeq sequencer and analyzed using a custom script to determine the signal

479 enrichment over background at TSSs over a $2 \mathrm{~kb}$ window.(

480 https://www.encodeproject.org/data-standards/atac-seq/) Only libraries that had

481 enrichment scores above 6 were sequenced deeper in a NextSeq Illumina sequencer.

\section{Cohesin HiChIP}

484 In situ chromosome conformation capture (3C) was performed as described earlier ${ }^{10}$.

485 Briefly, $25 \times 10^{6}$ cells were crosslinked and digested with Mbol (NEB). After digest, biotin 
was incorporated into the sticky ends of fragments before ligation. Cohesin ChIP was

487 performed to enrich for proximity ligations bound to cohesin, using an SMC1 antibody

488 (Bethyl, A300-055A). The library quality was assessed on a MiSeq sequencer before

489 sequencing on an Illumina HiSeq. Three replicates were pooled and sequenced across

490 two HiSeq lanes for a total of 1200 million reads per sample.

\section{UMI-4C}

493 UMI-4C was performed as described previously ${ }^{27}$. Briefly, $1 \times 10^{7}$ cells were crosslinked in 494 suspension with $1 \%$ formaldehyde then quenched with glycine, and pelleted cells were 495 lysed in $1 \mathrm{~mL}$ fresh cold lysis buffer (50 mM Tris- $\mathrm{HCl}, \mathrm{pH}$ 7.5, $150 \mathrm{mM} \mathrm{NaCl}, 5 \mathrm{mM}$

496 EDTA, 0.5\% NP-40, 1\% TX-100, 1x protease inhibitors) on ice. The nuclei were

497 extracted and resuspended in water, Dpnll buffer, and 10\% SDS for Dpnll digestion.

498 Three rounds of Dpnll digestion were performed, adding $200 \mathrm{U}$ of HC Dpnll (NEB) for 2

499 hours, incubating overnight, and then 2 more hours all at $37^{\circ} \mathrm{C}$ with rotation. After

500 inactivation of Dpnll, the $3 \mathrm{C}$ reactions were diluted to $7 \mathrm{~mL}$ and $13.6 \mathrm{uL}$ of $\mathrm{HC}$ T4 ligase

501 (NEB) were added for overnight ligation at $16^{\circ} \mathrm{C}$. Crosslinks were reversed overnight at

$50265^{\circ} \mathrm{C}$ with Proteinase K (Qiagen) and DNA was treated with RNase A (Qiagen) for 45

503 minutes at $37^{\circ} \mathrm{C}$. The DNA was then purified with one phenol-chloroform extraction

504 (ThermoScientific) and ethanol precipitation, and resuspended in $150 \mathrm{uL}$ of $10 \mathrm{mM}$ Tris-

$505 \mathrm{HCl}, \mathrm{pH}$ 8.0. DNA was quantified using Qubit before proceeding with library

506 preparations. Aliquots of chromatin were taken before and after Dpnll digestion and after

507 overnight ligation to determine efficiency of enzymatic reactions. UMI-4C library

508 preparation was performed as described previoiusly ${ }^{27}$. Briefly, 5-10 ug of 3C library was

509 sonicated in a Diagenode Bioruptor to achieve a chromatin size between 400 and 600

510 bp. End-repair (NEB kit), A-tailing (NEB kit) and 5'-dephosphorylation (NEB) of ends

511 were performed as recommended by the manufacturer. TruSeq Illumina indexed 
512 adapters were ligated to the 3'-end of the DNA using Quick Ligase (NEB). Libraries were

513 generated by nested PCR at particular genomic loci using GoTaq Hot Start Polymerase

514 (Promega) and 200 ng of DNA template (Extended Data Fig. 9). The primer for the

515 second PCR included the Illumina dangling adaptor for enrichment of the product from

516 the first PCR, as described ${ }^{27}$. Paired-end libraries were sequenced in the NextSeq

517 sequencer.

518

\section{Next Generation Sequencing processing of ChIP-seq and ATAC-seq data}

520 Quality control of fastq files was done with FASTQC ${ }^{32}$. Sequence alignment to hg19 was

521 performed using tophat for RNA-seq (parameters: p 10 --library-type fr-firststrand -r 100 -

522 -mate-std-dev 100), or bowtie2 ${ }^{33}$ for ChIP-seq (parameters: -p 24 -S -a -m 1 --best -

523 strata) and ATAC-seq (parameters: -p 24 -S -m 1 -X 2000). Aligned reads were

524 processed to remove PCR duplicates using samtools ${ }^{34}$ and mitochondrial DNA (for

525 ATAC-seq datasets). Peak calling was carried out with MACS2 ${ }^{35}$ using default settings

526 with a p-value of 0.05 . To filter out non-reproducible peaks, called peaks from biological

527 replicates were processed through the Irreproducible Discovery Rate (IDR) framework

528 implemented in $\mathrm{R}^{36}$.

\section{Differential counting, heatmaps, and average profiles}

531 For ChIP-seq and ATAC-seq, a union list of the MACS2 called peaks per sample not

532 filtered by IDR was generated using bedtools merge ${ }^{37}$, and raw reads covering each

533 region were recovered from bam files using bedtools multicoverage. For RNA-seq, raw

534 reads on reference genes were recovered using HOMER (version $4.8^{38}$

535 analyzeRepeats.pl command). To test for differential counting, raw reads were

536 compared using DESeq2 package implemented in $\mathrm{R}^{39}$, and filtered based on an

537 adjusted $p$-value of $<0.05$ and 2 fold change. For heatmaps and average profiles, tag 
538 counts were recovered +/- $2 \mathrm{~kb}$ from the peak summit using HOMER annotatePeaks.pl

539 command with -hist 25 -ghist or -hist 25 parameters. Heatmap images were generated

540 using Java TreeView ${ }^{40}$. Average profiles and scatter plots were plotted using Python

541 matplotlib.

542

\section{Motif discovery and Gene Ontology}

544 De novo motif discovery was performed using Homer findMotifsGenome function with 545 size 200 as a parameter. Gene ontology analysis was performed using DAVID ${ }^{41}$ for

546 RNA-seq data and GREAT ${ }^{42}$ for ChIP-seq and ATAC-seq data.

\section{Chromatin state determination}

549 ChromHMM software ${ }^{43}$ was used to learn and identify chromatin states as instructed in

550 the manual. Encode chromatin segmentation using ChromHMM was used as a

551 reference to label each state using a custom script using bedtools intersect. The

552 enrichment of each state for a set of peaks was calculated using the

553 NeighborhoodEnrichment command and compared among samples using a custom

554 script. Enrichments were plotted using Python matplotlib library.

556 Analysis of UMI-4C data

557 UMI-4C data was aligned and analyzed using $\mathrm{HiC}-\mathrm{Pro}^{44}$ and the Dpnll segmented

558 genome annotation file. Interaction matrices of $5 \mathrm{~kb}$ resolution were generated and used

559 to create Virtual 4C profiles through a custom python script and the matplotlib library.

\section{Analysis of cohesin HiChIP data}

562 HiChIP paired end reads were aligned to hg19 using HiC-Pro ${ }^{44}$. Duplicate reads were

563 removed, assigned to $\mathrm{Mbol}$ restriction fragments, filtered for valid interactions, and then 
564 used to generate binned interaction matrices of both $5 \mathrm{~kb}$ and $10 \mathrm{~kb}$ resolution. The $5 \mathrm{~kb}$

565 interaction matrices were used to visualize contacts by Virtual 4C, similar to the UMI-4C

566 analysis. The $10 \mathrm{~kb}$ interaction matrix was used to call high confidence contacts (defined

567 as counts $\geq 10$, FDR $<0.001$ ) using the contact caller, FitHiC ${ }^{23}$. Default FitHiC settings

568 were used to generate an FDR for each bin pair. These high confidence cohesin

569 contacts were used in the subsequent analyses.

570

\section{Contact connection analyses}

572 An element was considered participating, or anchored, in cohesin connections, if it

573 possessed at least one high confidence contact bin in a given cell type. When

574 considering ways in which p63 was connected to a TSS (defined as TSS +/- $5 \mathrm{~kb}$ ), four

575 chromatin conformations were considered. $0^{\circ}$ connections were defined as two elements

576 overlapping in physical space (e.g. p63 BS contained within the TSS). $1^{\circ}$ connections

577 were defined as one element anchored in one bin of a cohesin contact and the second

578 element anchored in the other bin. More complicated connections between elements

579 were also considered: $2^{\circ}$ connections were defined as two elements in distinct physical

580 space both forming $1^{\circ}$ connections to the same third element. Finally, $3^{\circ}$ connections

581 were when one element formed a $1^{\circ}$ connection to a second element, which also formed

582 a $1^{\circ}$ connection to a third element, which also had a $1^{\circ}$ connection to the fourth (target)

583 element. All elements in both $2^{\circ}$ and $3^{\circ}$ configurations were in distinct physical space

584 (i.e. non-overlapping).

\section{Differential contact analysis}

587 The Bioconductor package edgeR ${ }^{45}$ was used to perform multiple comparison differential 588 analysis of high confidence FitHiC contacts in d0, d0 p63GOF, d7 p63WT, and d7 589 p63KO cells. The Anderson-Darling K-sample test, a modification of the K-S test, which 
590 gives greater weight to the tails, was used to calculate statistical significance between

591 populations of the fold change in contact connectivity ${ }^{46}$.

592

593 Code Availability

594 Custom scripts described in the Methods will be made available upon request.

595

596 Data Availability

597 All sequencing data will be available through Gene Expression Omnibus (GEO) -

598 accession number pending.

599

600 A Life Sciences Reporting Summary for this publication is available.

601

602 
603

604

605

606

28. Hsu, P.D., et al. DNA targeting specificity of RNA-guided Cas9 nucleases. Nature biotechnology 31, 827-832 (2013).

29. Mali, P., Esvelt, K.M. \& Church, G.M. Cas9 as a versatile tool for engineering biology. Nature methods 10, 957-963 (2013).

30. Natsume, T., Kiyomitsu, T., Saga, Y. \& Kanemaki, M.T. Rapid Protein Depletion in Human Cells by Auxin-Inducible Degron Tagging with Short Homology Donors. Cell Rep 15, 210-218 (2016).

31. Buenrostro, J.D., Giresi, P.G., Zaba, L.C., Chang, H.Y. \& Greenleaf, W.J. Transposition of native chromatin for fast and sensitive epigenomic profiling of open chromatin, DNA-binding proteins and nucleosome position. Nature methods 10, 1213-1218 (2013).

32. Andrews, S. FastQC: a quality control tool for high throughput sequence data. . (2010).

33. Hwang, S., Kim, E., Lee, I. \& Marcotte, E.M. Systematic comparison of variant calling pipelines using gold standard personal exome variants. Sci Rep 5, 17875 (2015).

34. Li, H., et al. The Sequence Alignment/Map format and SAMtools. Bioinformatics 25, 2078-2079 (2009).

35. Zhang, Y., et al. Model-based analysis of ChIP-Seq (MACS). Genome biology 9 , R137 (2008).

36. Li, H. A statistical framework for SNP calling, mutation discovery, association mapping and population genetical parameter estimation from sequencing data. Bioinformatics 27, 2987-2993 (2011).

37. Quinlan, A.R. \& Hall, I.M. BEDTools: a flexible suite of utilities for comparing genomic features. Bioinformatics 26, 841-842 (2010). 
628 38. Heinz, S., et al. Simple combinations of lineage-determining transcription factors

629 prime cis-regulatory elements required for macrophage and B cell identities.

$630 \quad$ Molecular cell 38, 576-589 (2010).

631 39. Love, M.I., Huber, W. \& Anders, S. Moderated estimation of fold change and 632 dispersion for RNA-seq data with DESeq2. Genome biology 15, 550 (2014).

633 40. Saldanha, A.J. Java Treeview--extensible visualization of microarray data.

634 Bioinformatics 20, 3246-3248 (2004).

635 41. Huang da, W., Sherman, B.T. \& Lempicki, R.A. Systematic and integrative

636 analysis of large gene lists using DAVID bioinformatics resources. Nature

637 protocols 4, 44-57 (2009).

638 42. McLean, C.Y., et al. GREAT improves functional interpretation of cis-regulatory 639 regions. Nature biotechnology 28, 495-501 (2010).

640 43. Ernst, J. \& Kellis, M. ChromHMM: automating chromatin-state discovery and 641 characterization. Nature methods 9, 215-216 (2012).

642 44. Servant, N., et al. HiC-Pro: an optimized and flexible pipeline for Hi-C data 643 processing. Genome biology 16, 259 (2015).

644 45. Anders, S., et al. Count-based differential expression analysis of RNA 645 sequencing data using R and Bioconductor. Nature protocols 8, 1765-1786 646 (2013).

647 46. Scholz, F. \& Stephens, M. K-Sample Anderson-Darling Tests. J Am Stats Assoc $648 \quad$ 82, 918-924 (1987). 
bioRxiv preprint doi: https://doi.org/10.1101/348839; this version posted June 16, 2018. The copyright holder for this preprint (which was not certified by peer review) is the author/funder. All rights reserved. No reuse allowed without permission.

a

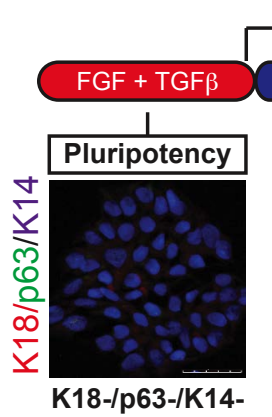

do

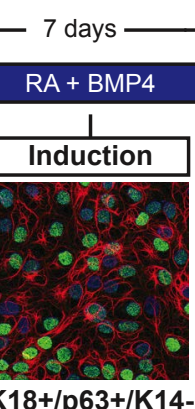

d7

d

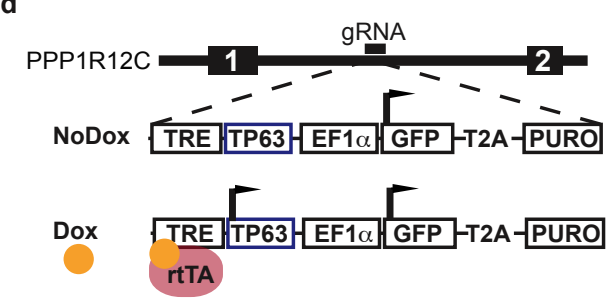

e

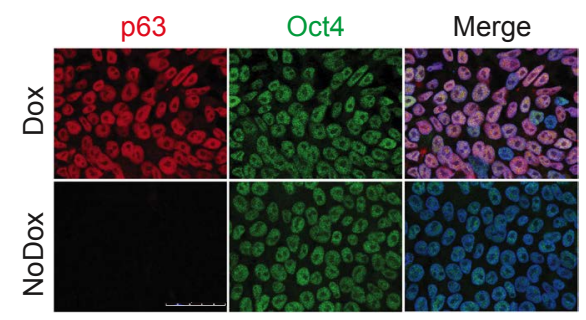

p63 gain of function at day 0

h
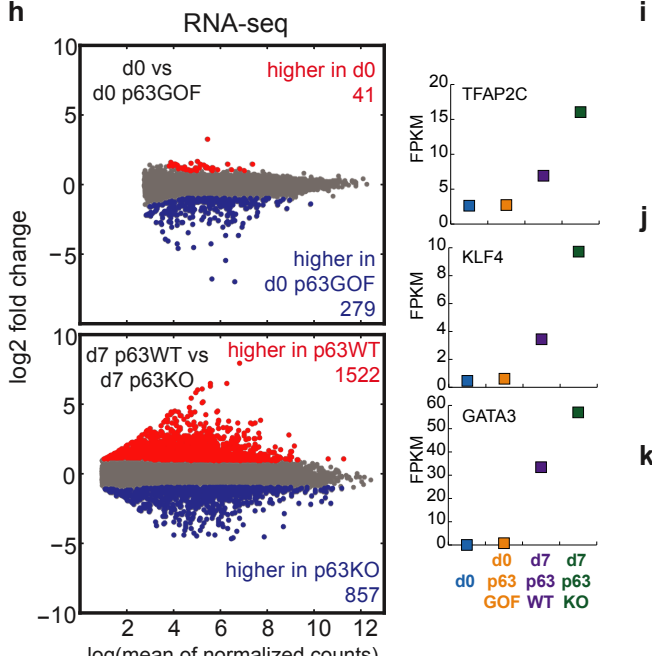

$45-60$ days Keratinocyte media

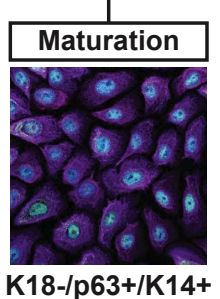

kc

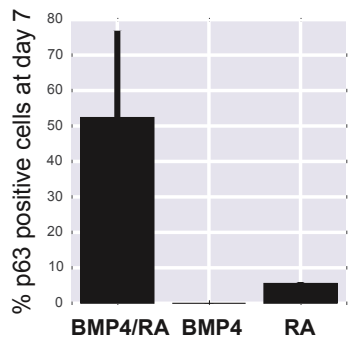

c

p63

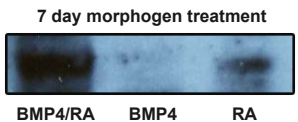

f

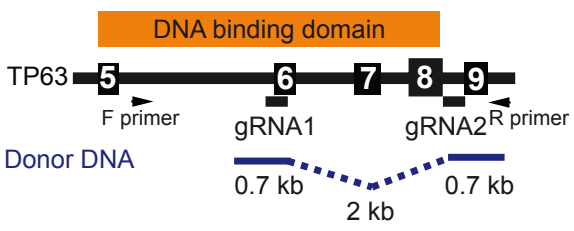

g

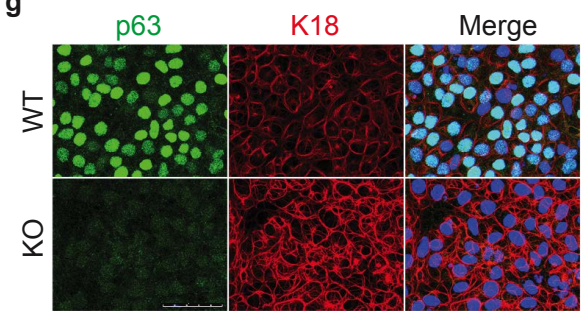

p63 loss of function at day 7 p63(p53)/Keratinocyte-p63 ChIP-Seq

p-val: AAGG ITCTCCAGACATCCC d0 p63GoF 1e-1644 GTCTCA

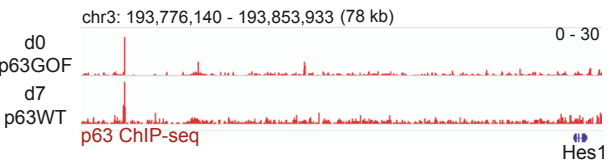

k

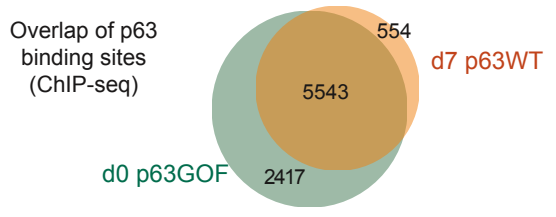

Figure 1. Morphogens and the lineage selector p63 cooperate to drive early stratified epithelial differentiation. (a) Differentiation of hESCs into keratinocytes takes 60 days in the xeno-free, defined system. Treatment with RA and BMP4 for 7 days induces K18 and p63 expression. Switching the cells into keratinocyte media allows for selection and growth of functional keratinocytes (kc) that express K14 and p63. (b,c) hESCs need exposure to both RA and BMP4 to achieve high p63 expression. Error bars represent standard deviation. (d) Strategy for generating the d0 p63GOF cell line. Numbered black boxes signify exons. (e) Expression of p63 in the d0 p63GOF line, showing even with Dox treatment, there is no loss in Oct4 expression. (f) Strategy for generating the p63KO line, using a two gRNA CRISPR/Cas9 approach. (g) IF validation of the p63KO line, showing loss of p63 expression and higher levels of K18. All IF scale bars represent $50 \mu \mathrm{m}$. (h) Differential expression analysis from RNA-seq (measured by DESeq2) between the d0 and d0 p63GOF lines (upper panel), and the d7 p63WT and p63KO lines (lower panel). The gray dots on the scatter plot represent no change in gene expression between the two cell types, while red represents increased expression in the d0 or $\mathrm{d} 7 \mathrm{wild}$ type by a $>2$ fold change and blue represents decreased expression in the $\mathrm{d} 0$ or $\mathrm{d} 7$ wild type by $\mathrm{a}<-2$ fold change. Key transcription factors associated with epithelial development are induced by the morphogens and repressed by p63 at d7. (i) The p63 motif was the most significantly recovered motif under p63 ChIP-seq peaks in d0 p63GOF and d7 p63WT cells. (j) p63 binds distal to TSSs as depicted at the HES1 locus (70 kb away) and to the same sites in d0 p63GOF and d7 p63WT. (k) p63 binds to similar sites genome-wide with and without morphogen presence, as depicted in the Venn Diagram. The majority of the $d 7 \mathrm{p} 63$ binding sites are fully recovered in the $d 0$ p63GOF line. 
bioRxiv preprint doi: https://doi.org/10.1101/348839; this version posted June 16, 2018. The copyright holder for this preprint (which was not certified by peer review) is the author/funder. All rights reserved. No reuse allowed without permission.

a

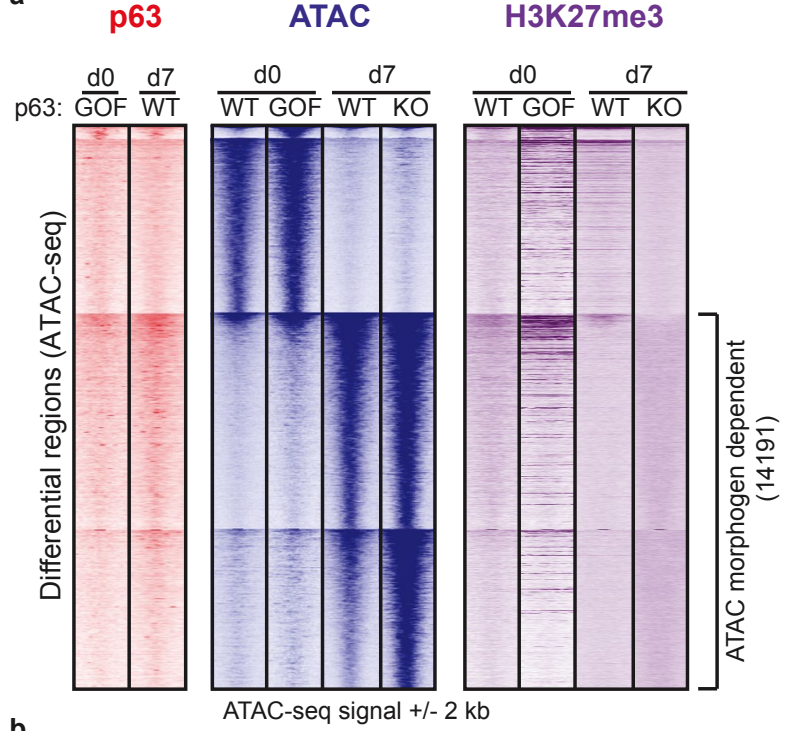

d

e

H3K27me3 ChIP-seq signal +/- $10 \mathrm{~kb}$
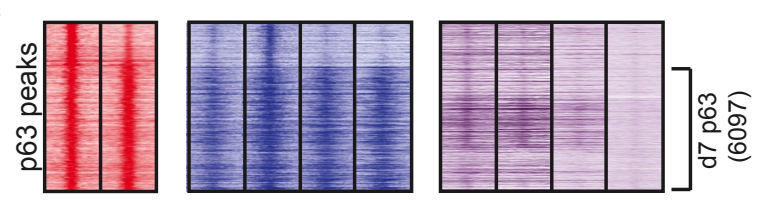

p63 ChIP-seq signal +/- $2 \mathrm{~kb}$

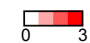

\begin{tabular}{ll}
\hline$\square$ & 3
\end{tabular}

$\underset{0 \quad 3.31}{\square+\square}$

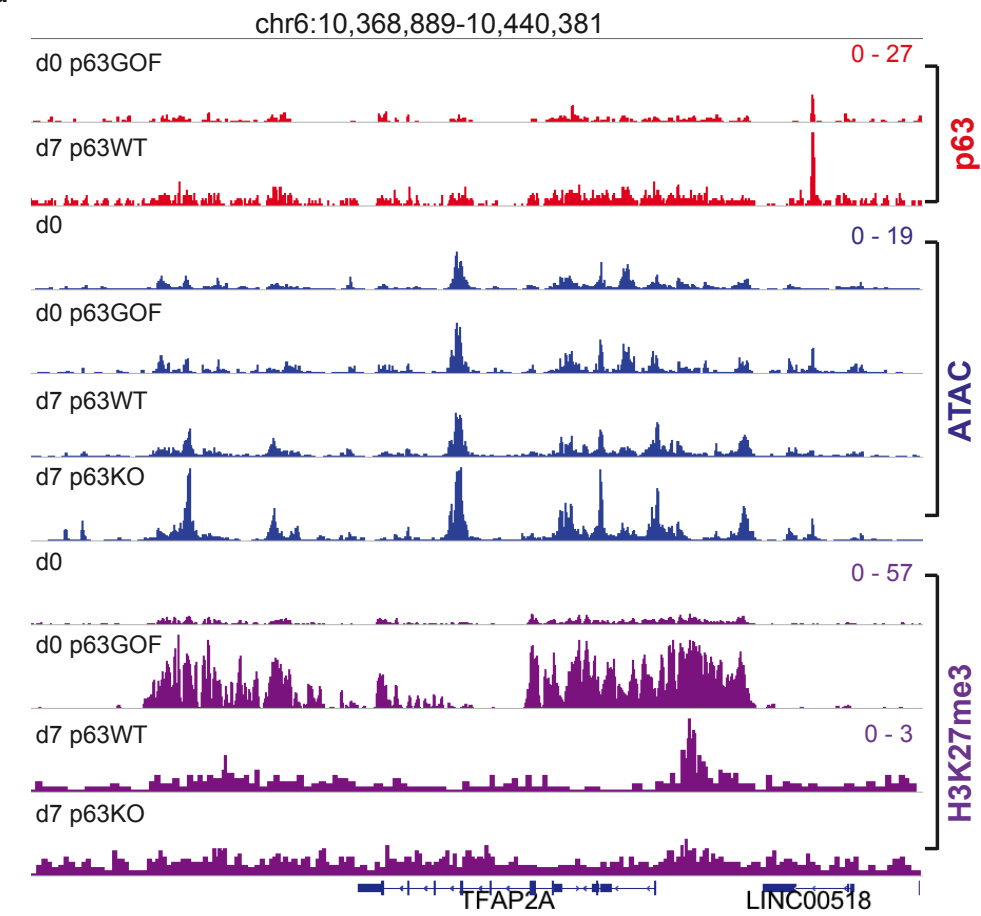

ஜำ

$\frac{4}{4}$

幽

f Intersect of genes linked to genomic locations by GREAT

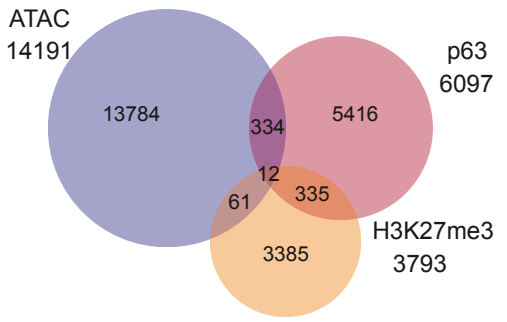

Figure 2. The morphogens establish an epigenetic landscape that p63 modifies at a distance. (a) Differential accessible regions between d0 and d7 p63WT as analyzed using DESeq2 on ATAC-seq signal. Heatmaps represent the signal at these ATAC regions within the various cell types and assays: p63 ChIP-seq signal (red, left panel), ATAC-seq signal (blue, middle panel), and H3K27me3 ChIP-seq signal (purple, right panel). 14,191 differential regions become more accessible upon morphogen treatment (morphogen-dependent). (b) Differential H3K27me3 regions between d7 p63WT and p63KO as analyzed by DESeq2. Heatmaps represent the same datasets as (a) only signal is shown at differential H3K27me3 sites (3,793 sites). (c) ATAC-seq (blue) and H3K27me3 (purple) signal at p63 binding sites (red). (d) Signal intensities of p63 ChIP-seq, ATAC-seq, and H3K27me3 ChIP-seq shown at the TFAP2A locus. (e) The overlap of genomic regions that are differential as measured in (a), (b), and (c). The genomic location intersect is very low. (f) GREAT analysis linking the above differential regions to the closest gene shows that these elements converge on a similar gene set. While their physical genomic locations do not overlap, they are linked to a common gene via GREAT. 
bioRxiv preprint doi: https://doi.org/10.1101/348839; this version posted June 16, 2018. The copyright holder for this preprint (which was not certified by peer review) is the author/funder. All rights reserved. No reuse allowed without permission.

a

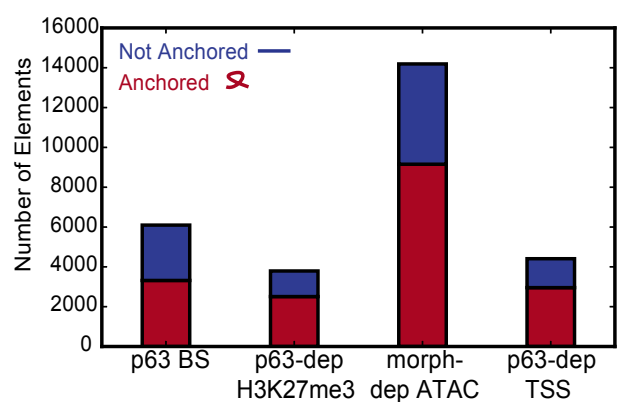

C

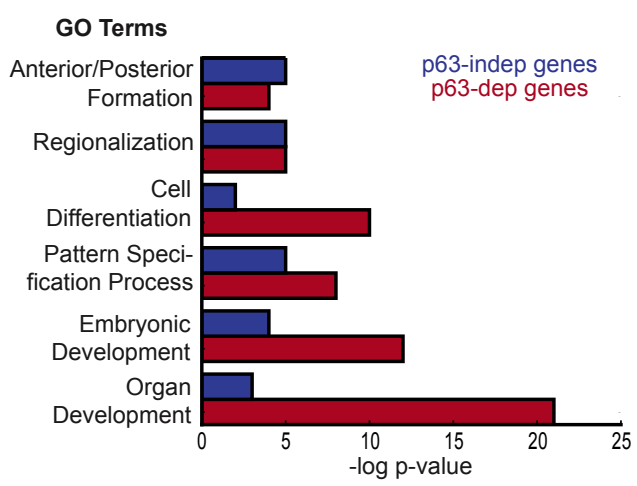

b

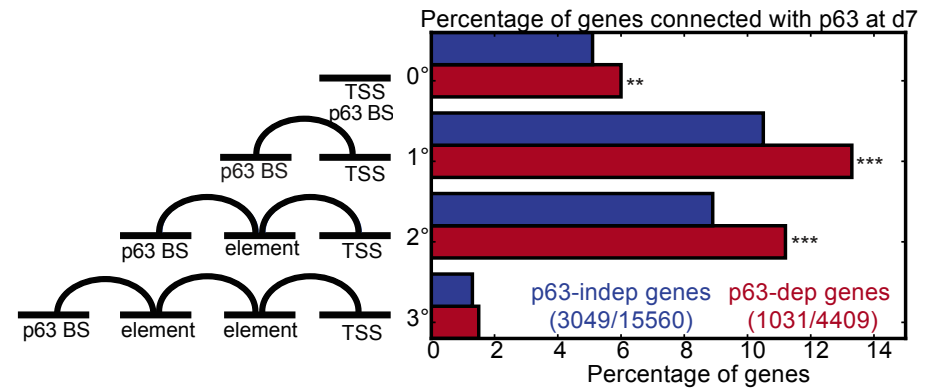

e

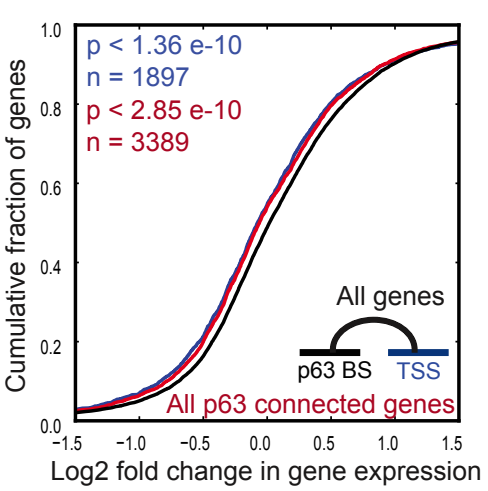

d7 p63WT elements connected by cohesin contacts

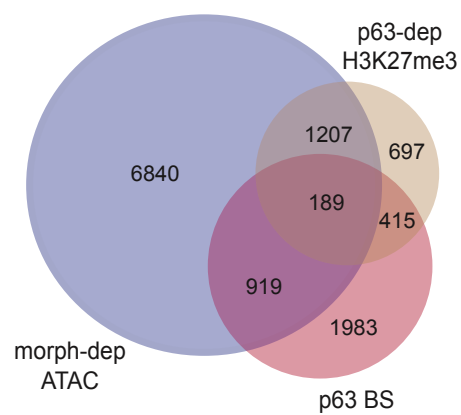

$\mathbf{f}$

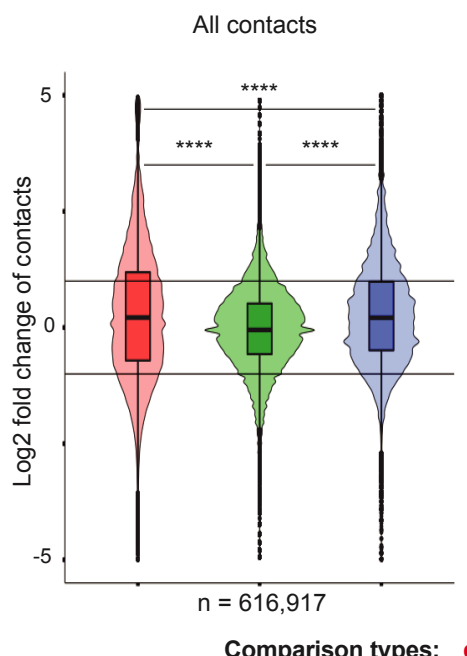

d7 p63WT p63 BS morph-

dep-ATAC

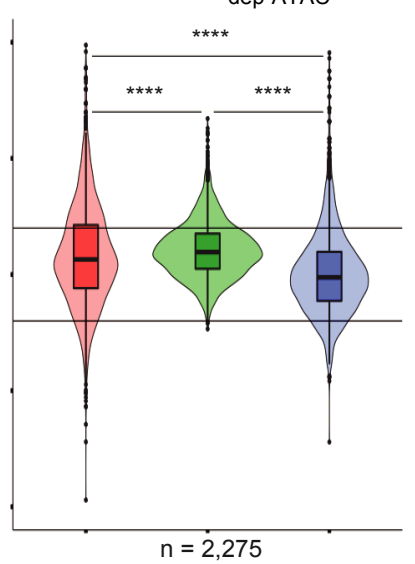

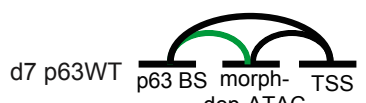

dep-ATAC

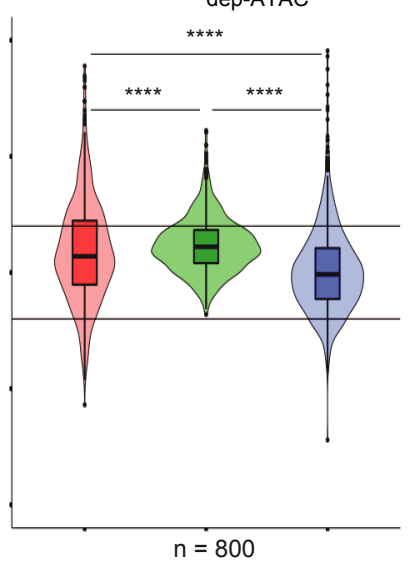

g

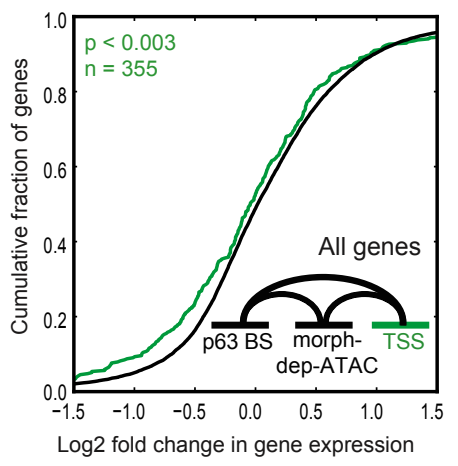

d0 vs d7 p63WT d7 p63WT vs d7 p63KO d0 vs d0 p63GOF

Figure 3. p63 - TSS connections are associated with negative regulation genome-wide. (a) Number of p63 binding sites (BS), p63-dependent (p63-dep) H3K27me3 sites, morphogen-dependent (morph-dep) ATAC sites, and p63-dependent TSSs that participate in chromatin looping (Anchored, red) vs those that do not (Not Anchored, blue) in d7 p63WT cells. (b) Percentage of p63-independent (p63-indep) genes (blue) and p63-dep genes (red), whose TSS is connected to p63 by direct binding $\left(0^{\circ}\right)$, direct contact $\left(1^{\circ}\right)$, or connected via one $\left(2^{\circ}\right)$ or two $\left(3^{\circ}\right)$ morph-dep ATAC and/or p63-dep H3K27me3 elements. (c) Gene Ontological Terms associated with p63-indep genes (blue) and p63-dep genes (red), which are connected to p63. (d) ecdf of the log2 fold change in gene expression between d7 p63WT vs d7 p63KO cells (d7 p63KO / d7 p63WT) for all p63 connected genes (red) and p63 $1^{\circ}$ connected genes (blue) compared to all genes (black). (e) $1^{\circ}$ contact connections between p63 BS (red), p63-dep H3K27me3 (gold), and morph-dep ATAC (blue). (f) Change in connectivity strength between various cell types of all contacts (left panel), p63 morph-dep ATAC contacts (middle panel), and p63 - morph-dep ATAC contacts in which both elements are connected to the TSS (right panel). $\mathrm{n}=$ number of contacts. ( $\mathrm{g}$ ) ecdf of the change in expression level (d7 p63KO / d7 p63WT) of genes whose TSS is connected to a p63 BS and morph-dep ATAC site, which in turn are connected to each other (green) compared to all genes (black). FDR by monte carlo simulation ${ }^{* *} \mathrm{FDR}<0.01,{ }^{* * *} \mathrm{~F}$ $\mathrm{DR}<0.001$. Angela-Darling k-samples test ${ }^{* * *} \mathrm{P}<1 \times 10^{-10}$. 
bioRxiv preprint doi: https://doi.org/10.1101/348839; this version posted June 16, 2018. The copyright holder for this preprint (which was not certified by peer review) is the author/funder. All rights reserved. No reuse allowed without permission.

a
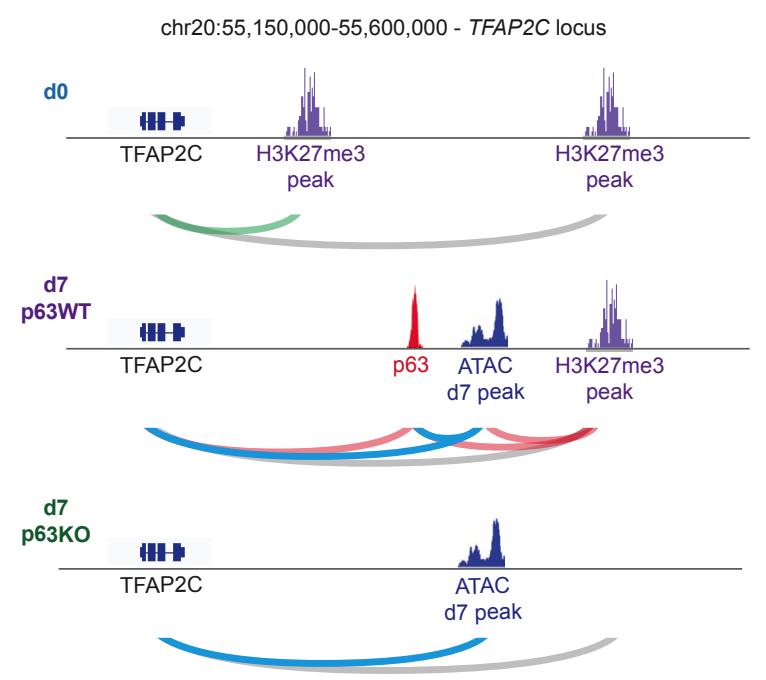

Looping interaction type:

stable hESC-specific p63-dependent morphogen-dependent
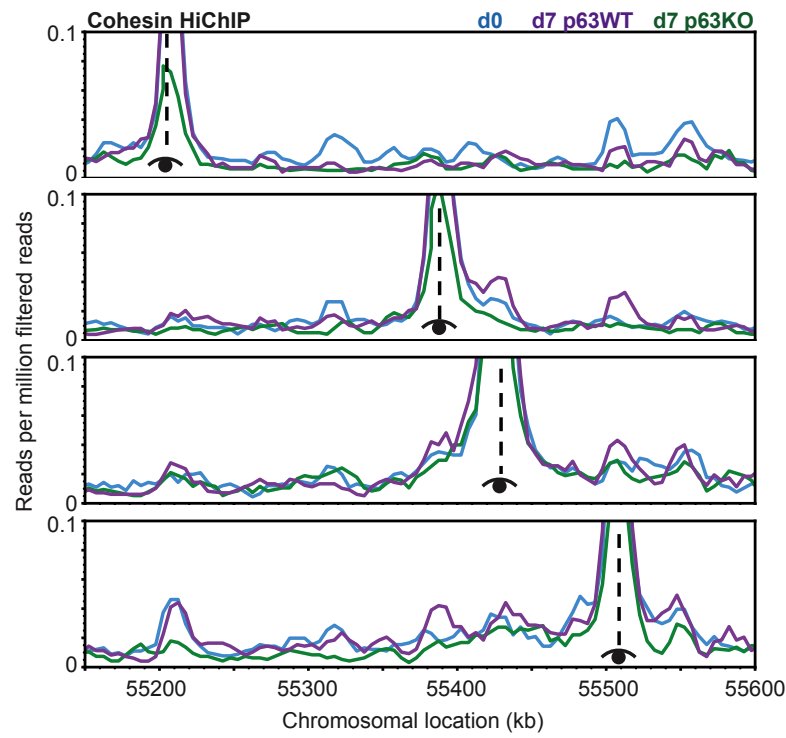

b

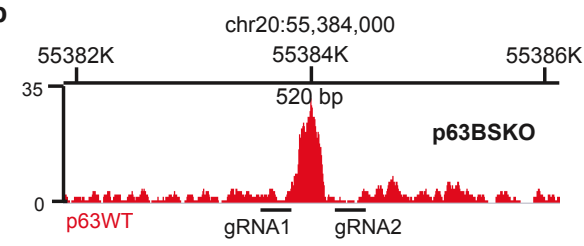

C

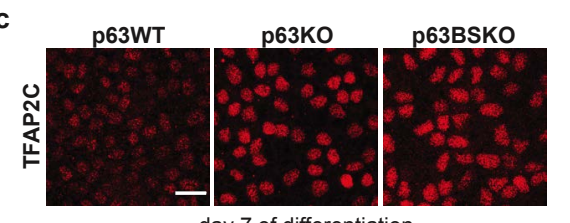

day 7 of differentiation

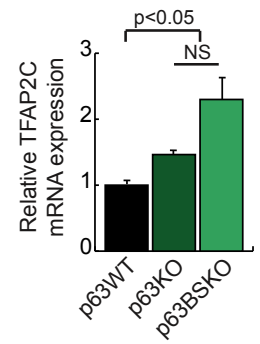

d
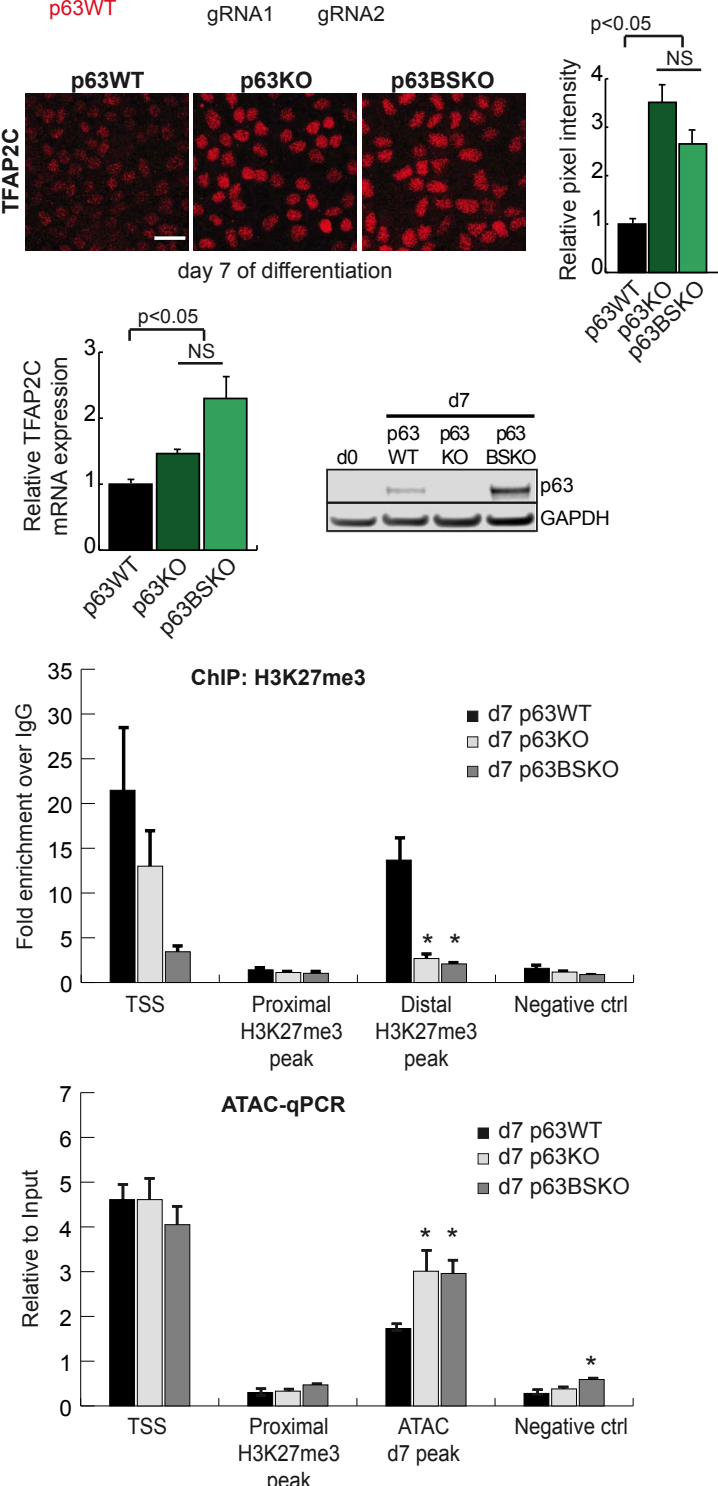

peak

Figure 4. p63 negatively regulates TFAP2C expression through morphogen-induced and p63-dependent distal elements and connectivity. (a) Cohesin HiChIP reveals complex looping interactions between numerous distal elements at the TFAP2C locus. Schematic for the morphogen and p63-dependent interactions (top panel) with virtual 4C plots of the normalized cohesin HiChIP data below (bottom panel). (b) The p63 binding site was deleted using CRISPR/Cas9 to determine the effects of its loss on TFAP2C expression. p63BSKO is a 520 bp deletion surrounding the p63 ChIP-seq peak. (c) Deletion of the p63 binding site leads to an increase in TFAP2C expression similar to the levels seen in the d7 p63KO cells (NS - not significant). Loss of TFAP2C expression also leads to a dramatic increase in p63 expression. Relative pixel intensity was calculated from 3 independent images and scale bars represent $20 \mu \mathrm{m}$. (d) ChIP-qPCR for H3K27me3 at the TFAP2C locus shows a decrease in the histone mark in the $d 7 \mathrm{p} 63 \mathrm{BSKO}$ cells, similar to the $\mathrm{d} 7 \mathrm{p} 63 \mathrm{KO}$ cells ( ${ }^{*} \mathrm{p}$-value $<0.01$ ). ATAC-qPCR at this locus shows an increase in accessibility at the $d 7$ ATAC peak in $d 7$ p63BSKO, again similar to the $d 7$ p63KO ( ${ }^{*} p$-value $<0.05$ ). Deletion of the $p 63$ binding site results in a loss of tightly controlled TFAP2C expression. Both graphs depict signal relative to input and error bars represent standard error of the mean. 